Research Article

Xin $\mathrm{Li}^{\star}$ and Yang Wang

\title{
Evaluation of AHRS algorithms for Foot-Mounted Inertial-based Indoor Navigation Systems
}

https://doi.org/10.1515/geo-2019-0005

Received May 16, 2018; accepted Jul 24, 2018

\begin{abstract}
Personal Dead Reckoning based on footmounted Inertial Measurement Units is a research hotspot in the field of positioning and navigation in recent years. This paper conducts a targeted research on the application of current mainstream attitude and heading reference system (AHRS) algorithm in the foot inertial navigation positioning. Through open datasets, the positioning accuracy and directional accuracy of 9-state complementary Kalman filter (CKF) are compared and analyzed among the conventional algorithm, Mahony algorithm, and Madgwick algorithm, in which the Madgwick algorithm can achieve the best positioning results. And on this basis, for the Madgwick algorithm, it is verified that it can help improve the positioning accuracy of 15 -state CKF under the assistive technologies of zero angular rate update (ZARU) and heuristic heading reduction (HDR). The adaptive zerospeed detection algorithm is designed, and the threshold value of zero-speed detection is set dynamically through tracking the variable of speed in CKF, which can detect the time period of zero-speed state more accurately, thus further improving the correction of directional errors. Finally, the effectiveness of the proposed algorithm is further proved by actual data.
\end{abstract}

Keywords: Foot-mounted IMU; AHRS; Complementary Kalman filter; Mahony; Madgwick

\section{Introduction}

Pedestrian indoor navigation systems constructed around foot-mounted inertial measurement units (IMUs) have shown promising results [1-3]. In order to overcome the accumulation of errors, the Zero Velocity Update (ZUPT)

\footnotetext{
^Corresponding Author: Xin Li: School of Computer Science and Technology, China University of Mining and Technology, Xuzhou 221116, China; Email: linuxcumt@126.com

Yang Wang: School of Computer Science and Technology, China University of Mining and Technology, Xuzhou 221116, China
}

is generally used to modify the system error. This method was first proposed by E. Foxlin [4] in 2005 and applied in the NavShoe project. Since then, a large number of studies on this kind of foot-mounted indoor inertial navigation and positioning system have appeared, of which, some successful ones are as follows.

A double-layer Bayesian position estimation framework based on foot inertial sensors was proposed by Robertson et al. [5] in 2008, in which, the Kalman filter and the particle filter was used in the bottom layer and the top layer, respectively. In 2009, a new Personal Dead Reckoning (PDR) technology called FootSLAM was created based on the Simultaneous Localization and Mapping (SLAM) algorithm, and the particle filter algorithm was used to correct the trajectory data acquired previously [6]. In 2010, the PlaceSLAM, a system for pedestrians to upload maps online, was provided by them. Besides, a set of reference datasets was also provided, and the positioning accuracy was verified by using optical positioning system with high accuracy [7]. In 2011, based on FootSLAM and PlaceSLAM, the Wi-Fi support for the system was introduced and a large-scale map was constructed by iterative calculations, named WiSLAM [8]. In 2013, map information for some large sites was successfully generated by the improved FootSLAM program [9].

An open-source and real-time inertial navigation system based on foot-mounted ZUPT was proposed in 2012 by Nilsson, Skog et al. [10] from the Signal Processing Laboratory of the Swedish Royal Institute of Technology. The cost of samples was about 800 US dollars, and the navigation error range was $0.2 \%-1 \%$ within a short distance which was less than 100 meters. At the same time, the limitations and the error model of ZUPT were also analyzed [11], and the algorithm robustness was enhanced by eliminating drift errors with an optimized algorithm [12]. In 2013, a positioning algorithm based on double foot-mounted inertial sensors was proposed [13]. For this algorithm, the distance between the two feet was used to constrain the heading angle offset of the inertial devices, which greatly improved the accuracy of autonomous positioning. In 2014, a positioning method based on multiple-IMU was developed by the Nilsson team [14], which could further improve the reli-

○ Open Access. (C) 2019 X. Li and Y. Wang, published by De Gruyter. (Cc) BY 4.0 License

This work is licensed under the Creative Commons Attribution 4.0 License 
ability and accuracy of autonomous positioning, besides, its positioning experiment platform was open-sourced. In 2016, the estimation formula and analysis of MIMU systems were presented by Nilsson et al. [15]. The formulation makes it possible to analyze the fundamental properties of such systems and to derive efficient estimators.

The main factor that reduces performance of the footmounted PDR is an error in estimation of the sensor orientation [16]. In particular, the yaw, which represents the heading error, is more critical. The accumulation of the heading error will cause a large shift in the overall trajectory even if the integral error of each step is small. Although ZUPT can indirectly compensate the position error and heading error by correcting velocity, it still has four disadvantages as followings $[17,18]$. First, the error can be corrected by ZUPT only when the velocity is close to zero. Second, only the roll and the pitch angles can be corrected by ZUPT, while the yaw angle cannot be. Third, Zero-velocity hypothesis in some cases can be incorrect due to the small movements in zero-velocity-phase, which in the end could lead to modelling error. Forth, due to the different features of different gait such as running, climbing and etc., a uniform zero-velocity detector cannot correctly detect all the zero velocity situations, and as a result, a zero velocity maybe missed detected or an effective velocity maybe incorrectly set to zero.

How to further improve the accuracy of direction computation in the foot-mounted PDR algorithm is the focus of this paper. Gyroscopes in the IMU can calculate the direction by integral; however, the prolonged integral data is liable to drift, thus causing a big directional error. Accelerometers and magnetometers can also be used to calculate the direction, but the data obtained from them are especially vulnerable to noisy data, thus causing a big error in the direction. state-of-art Attitude and Heading Reference Systems(AHRS), such as Kalman filter, Mahony, Madgwick and etc., combines the accelerometer, gyroscope and magnetometer measurements with two absolute fields, the Earth magnetic field and the gravity field, whose directions and intensities are known, help estimating the orientation. They have made full use of the longterm reliability of accelerometers and magnetometers in calculating the direction and the short-term reliability of gyroscope data, thus generating the direction computation results with higher accuracy.

Therefore, several state-of-art Attitude and Heading Reference Systems (AHRS) such as common algorithm, Mahony [19] and Madgwick [20] are introduced to the footmounted PDR algorithm in this paper, as shown in Figure 1. The pink box in Figure 1 is the Complementary Kalman Filter(CKF) algorithm [21] used to estimate the po- sition, velocity and direction deviations during the PDR. The calculation errors in attitude, direction and position can be effectively eliminated by substituting the above deviations into the INS Navigation Equation. In addition, the key of the CKF algorithm is the conditional constraints on the state variables, and the Magnetic Angular Rate Update (MARU) [22], Zero Angular Rate Update (ZARU) [23], Heuristic Heading Reduction (HDR) [23] and ZUPT are commonly used.

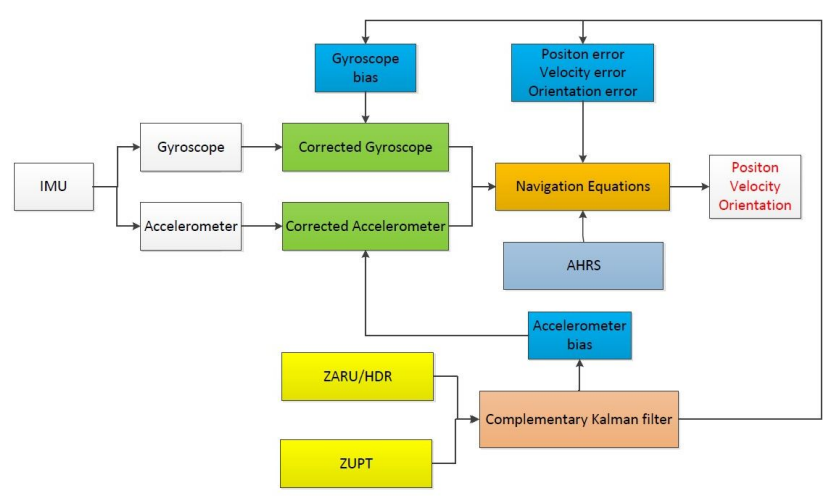

Figure 1: Algorithm flowchart

The main work of this paper includes:

1. The application of foot-mounted PDR algorithm is summarized through the comparison of 9-state CKF algorithm and AHRS algorithm. The Madgwick algorithm has higher accuracy because it has higher accuracy in calculating heading.

2. The AHRS algorithm is introduced based on the original computing framework of foot-mounted PDR algorithm. The advantage of using AHRS algorithm is that it can compensate attitude errors continuously instead of only at the stage of ZUPT, thus improving the directional accuracy on the whole.

3. As for the ZUPT algorithm, the dynamic setup of zero-speed detection threshold is proposed by tracking the velocity change of pedestrians, which improves the accuracy of zero-speed detection and can detect the time period of zero-speed state more accurately, thus further improving the correction of directional errors and reducing the positioning deviation.

The remainder of the paper is organized as follows. In Section 2, foot-mounted IMU positioning algorithm based on CKF is discussed, and the method of introducing AHRS algorithm to CKF is presented. In Section 3, the principle of the mainstream AHRS algorithm is discussed. Sub- 
sequently, several experiments are analysed in Section 4. Section 5 concludes the paper.

\section{Inertial Navigation and Zero-Velocity Updating}

\subsection{Introduction of AHRS algorithm to dynamic model}

Following the method in previous research [24], the nine state of the CKF algorithm is defined as $X=\left[\delta p^{n} \delta v^{n} \epsilon_{a}\right]$, where $\delta p^{n}$ is the position error, $\delta v^{n}$ is the velocity error, $\epsilon_{a}$ is the direction error. The state vector is under the navigation frame (n-frame). The direction, velocity and position under $\mathrm{n}$-frame can be obtained by integrating the gyroscope and acceleration data. The INS navigation equation is defined as:

$$
\begin{gathered}
\dot{p}^{n}=v^{n} \\
\dot{v}^{n}=C_{b}^{n} f^{b}+g^{n} \\
\dot{C}_{b}^{n}=C_{b}^{n}\left[\omega^{b} \times\right]
\end{gathered}
$$

where $g^{n}$ is the gravity vector under $\mathrm{n}$-frame, $f^{b}=$ $\left[f_{x}^{b}, f_{y}^{b}, f_{z}^{b}\right]$ is the acceleration vector under b-frame, $\omega^{b}=$ $\left[\omega_{x}^{b}, \omega_{y}^{b}, \omega_{z}^{b}\right]$ is the angular velocity under the body frame (b-frame), $\left[\omega^{b} \times\right]$ is the skew-symmetric matrix of angular velocity, defined as:

$$
\left[\omega^{b} \times\right]=\left[\begin{array}{ccc}
0 & -\omega_{z}^{b} & \omega_{y}^{b} \\
\omega_{z}^{b} & 0 & -\omega_{x}^{b} \\
-\omega_{y}^{b} & \omega_{x}^{b} & 0
\end{array}\right]
$$

For the acceleration and the observation of the gyroscope at moment $\mathrm{k}$, the drifts are required to be removed first, as shown in the following formula:

$$
\begin{aligned}
& \hat{f}_{k}^{b}=\tilde{f}_{k}^{b}-b_{a} \\
& \hat{\omega}_{k}^{b}=\tilde{\omega}_{k}^{b}-b_{g}
\end{aligned}
$$

$\tilde{f}_{k}^{b}$ and $\tilde{\omega}_{k}^{b}$ are original observations of acceleration and angular velocity, respectively; $\hat{f}_{k}^{b}$ and $\hat{\omega}_{k}^{b}$ are the corresponding values after deviation compensation. $b_{g}$ is the drift of the gyroscope; $b_{a}$ is the drift of the acceleration. For the CKF under State-9, these two values are obtained by the IMU calibration and used as constants to correct $\tilde{f}_{k}^{b}$ and $\tilde{\omega}_{k}^{b}$. For the CKF under State-15, these two values are the dynamic state variables of the system.

The transformation of the acceleration from Moment $\mathrm{k}$ to Moment $(\mathrm{k}+1)$ is:

$$
\hat{f}_{k+1}^{n}=C_{b}^{n}\left(\tilde{f}_{k}^{b}+0.5\left(\tilde{\omega}_{k}^{b} d_{t} \otimes \tilde{f}_{k}^{b}\right)\right)-g^{n}
$$

$\otimes$ denotes vector cross-product, representing the rotation correction of acceleration with change in angular velocity.

The transformation of the velocity from moment $k$ to moment $(\mathrm{k}+1)$ is:

$$
v_{k+1}^{n}=v_{k}^{n}+\hat{f}_{k+1}^{n} d_{t}
$$

The transformation of the position from moment $\mathrm{k}$ to moment $(\mathrm{k}+1)$ is:

$$
p_{k+1}^{n}=p_{k}^{n}+0.5\left(v_{k}^{n}+v_{k+1}^{n}\right) d_{t}
$$

The transformation of the attitude from moment $\mathrm{k}$ to moment $(\mathrm{k}+1)$ is:

$$
C_{b, k+1}^{n}=C_{b, k}^{n}\left(I+\left[\tilde{\omega}_{k}^{b} d_{t} \times\right]\right)
$$

$C_{b, k+1}^{n}$ is the rotation matrix at moment $(\mathrm{k}+1)$, with its value representing the compensated rotation of the pose change matrix from moment $\mathrm{k}$ to moment $(\mathrm{k}+1)$. In order to improve the accuracy of the attitude, the value of $C_{b, k+1}^{n}$ needs periodic normalization.

Equation 10 presents the AHRS Fundamental Approach. Our idea is to adopt Mahony or Madgwick algorithm instead of Equation 10 to calculate the attitude. Here, two points are worthy to be noted. First, the realization of CKF in [24] is based on the rotation matrix, while Mahony and Madgwick are based on the quaternion to calculate the attitude, so the calculated attitude results of each Mahony or Madgwick algorithm are required to be converted into rotation matrix. Second, the bias correction is already included in the Mahony and Madgwick algorithms, so it is unnecessary to correct the original accelerometer and gyroscope data in according to Equation 5 and 6.

\subsection{Zero-Velocity Detectors}

If a zero-velocity state is detected, the error can be calculated by the Kalman gain $K_{k}$ and the observed zero-velocity deviation $V_{k}$ :

$$
X_{k}=\left(\delta p^{n} \delta v^{n} \epsilon_{a}\right)=K_{k} V_{k}
$$

The position error, velocity error and direction error are contained in the vector $X_{k}$. Regarding $\epsilon_{a}$, a skew- 
symmetric matrix $\Omega_{\epsilon, k}$ for the angular error is constructed:

$$
\Omega_{\epsilon, k}=\left[\begin{array}{ccc}
0 & \epsilon_{a}[3] & -\epsilon_{a}[2] \\
-\epsilon_{a}[3] & 0 & \epsilon_{a}[1] \\
\epsilon_{a}[2] & -\epsilon_{a}[1] & 0
\end{array}\right]
$$

The attitude, velocity and position are corrected as follows:

$$
\begin{gathered}
C_{b, k}^{n}=\left(I-\Omega_{\epsilon, k}\right) C_{b, k}^{n} \\
v_{k}^{n}=v_{k}^{n}-\epsilon_{v} \\
p_{k}^{n}=p_{k}^{n}-\epsilon_{p}
\end{gathered}
$$

It should be noted that since the one used in the Mahony or Madgwick algorithm is quaternion, the corrected $C_{b, k}^{n}$ is required to be converted to a quaternion again for the next attitude calculation.

\section{Attitude and Heading Estimation Methods}

\subsection{AHRS Fundamental Approach}

The principles of attitude calculation are as follows: when expressing a certain vector $\mathrm{n}$ by using different coordinate systems, the size and direction must be the same. However, as there are some errors in the transformation matrix of these two coordinate systems, when a vector is transformed by a rotation matrix with some errors, it must be some deviations compared with the theoretical value in another coordinate system. And the attitude is corrected just by the correction of this rotation matrix through these deviations.

Since the rotation matrix is actually expressed by quaternions in this paper, the goal of attitude calculation is achieved by correcting the quaternions, and accelerometers and magnetometers are a main measurement object for attitude calculation.

Initially, assume that the sensor is either at a stand still or moves at a constant velocity so that only the gravity vector can be measured by the accelerometer. At the same time, assume a non-disturbed magnetic field and therefore the only the Earth magnetic field is measured by magnetometers. The $\operatorname{pitch}(\theta)$ and $\operatorname{roll}(\phi)$ can be calculated from the gravity vector, as shown in the following equation:

$$
\phi=\tan \left(f_{y}^{b} / f_{z}^{b}\right)^{-1}
$$

$$
\theta=-\sin \left(f_{x}^{b} / g\right)^{-1}
$$

The value of yaw $(\psi)$ can be obtained by the magnetometer instead of the gravity vector. The measurement of magnetometer, denoted as $m^{b}=\left[m_{x}^{b}, m_{y}^{b}, m_{z}^{b}\right]$, represents the value of the geomagnetic field under $b$-frame. Since pitch $(\theta)$ and roll $(\phi)$ are known, $m^{b}$ can be converted to the XOY plane under the $\mathrm{n}$-frame, and its value is denoted as $m^{n}=\left[m_{x}^{n}, m_{y}^{n}, m_{z}^{n}\right]$. The conversion relationship between the two groups of magnetic data is as follows:

$\left[\begin{array}{l}m_{x}^{n} \\ m_{y}^{n} \\ m_{z}^{n}\end{array}\right]=$

$\left[\begin{array}{ccc}\cos (\theta) & 0 & -\sin (\theta) \\ 0 & 1 & 0 \\ \operatorname{in}(\theta) & 0 & \cos (\theta)\end{array}\right]^{-1}\left[\begin{array}{ccc}1 & 0 & 0) \\ 0 & \cos (\phi) & 0 \\ 0 & 0 & \cos (\phi)\end{array}\right]^{-1}\left[\begin{array}{l}m_{x}^{b} \\ m_{y}^{b} \\ m_{z}^{b}\end{array}\right]$

Under the geographic coordinate system, the data of the geomagnetic field is $b=\left[b_{x}, 0, b_{z}\right]$, where $b_{y}$ in the east direction is 0 since the $b_{x}$ direction points to the north under the geographic system. In fact, the $\mathrm{n}$-frame and the geographic coordinate system are in a same XOY plane, and the difference angle happens to be the heading angle $\psi$, as shown in Figure 2. Among them,

$$
b_{x}=\operatorname{sqrt}\left[\left(m_{x}^{b}\right)^{2}+\left(m_{y}^{b}\right)^{2}\right]
$$

$$
b_{z}=m_{z}^{n}
$$

The consistent XOY plane is provided by the correction of accelerometers, that is, navigation coordinate system and geographic coordinate system are in one plane.

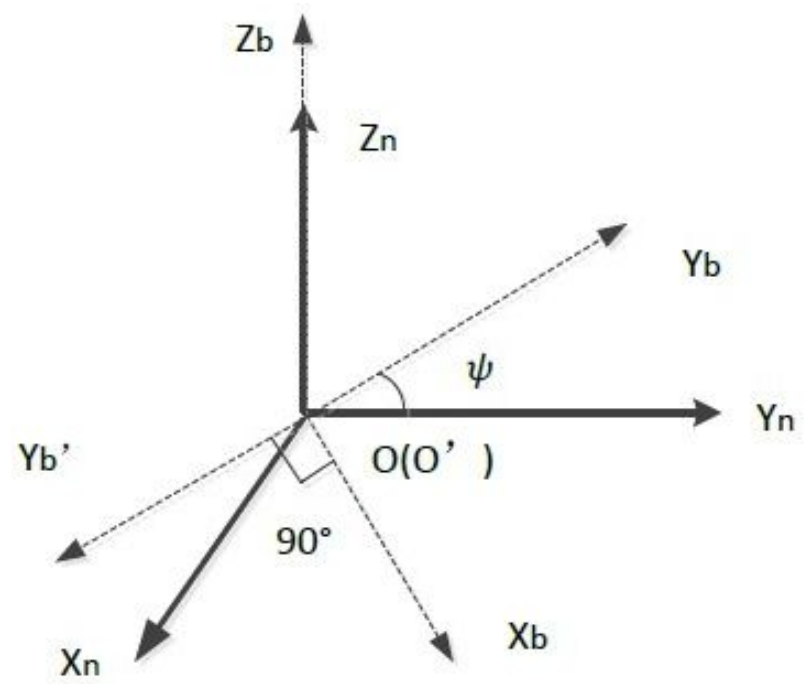

Figure 2: Navigation coordinate system and geographic coordinate system. 
Only by this way can Equation 19 be satisfied according to the relationship of trigonometric function.

The relationship between the data $b$ of the geomagnetic field and the magnetic field under the $b$-frame is as follows:

$$
b=C_{b}^{n} m^{b}
$$

The heading angle can be calculated by Equation 21:

$$
\begin{aligned}
& \psi= \\
& \arctan \left(\frac{m_{x}^{b} \cos (\phi)+m_{z}^{b} \sin (\phi)}{\left[m_{x}^{b} \sin (\phi)-m_{z}^{b} \cos (\phi)\right] \sin (\theta)-m_{y}^{b} \cos (\theta)}\right)
\end{aligned}
$$

After the initial yaw, pitch and roll are obtained, the attitude transition matrix $C_{b, 1}^{n}$ can be constructed as follows:

$$
\begin{aligned}
& {\left[\begin{array}{l}
\cos (\theta) \star \cos (\psi) \\
\cos (\theta) \star \sin (\psi) \\
-\sin (\theta)
\end{array}\right.} \\
& \sin (\phi)^{\star} \sin (\theta) \star \cos (\psi)-\cos (\phi)^{\star} \sin (\psi) \\
& \sin (\phi)^{\star} \sin (\theta){ }^{\star} \sin (\psi)+\cos (\phi)^{\star} \cos (\psi) \\
& \sin (\phi)^{\star} \cos (\theta) \\
& \cos (\phi)^{\star} \sin (\theta)^{\star} \cos (\psi)+\sin (\phi)^{\star} \sin (\psi) \\
& \cos (\phi)^{\star} \sin (\theta)^{\star} \sin (\psi)-\sin (\phi)^{\star} \cos (\psi) \\
& \cos (\phi)^{\star} \cos (\theta)
\end{aligned}
$$

The subsequent attitude transition matrix $C_{b, k+1}^{n}$ can be obtained by Equation 10 .

\subsection{Madgwick AHRS}

For the Madgwick algorithm, the current pose is calculated in the form of quaternion using a gyroscope:

$$
q_{t+1}=q_{t}+0.5 q_{t} \omega_{t} d_{t}
$$

By calculating the difference of accelerometer as well as the difference of magnetometer between $\mathrm{n}$-frame and $\mathrm{b}$ frame, the error of pose is corrected. Denote the vector coordinate under the $\mathrm{n}$-frame and the $\mathrm{b}$-frame as $e=$ $\left[0, e_{x}, e_{y}, e_{z}\right]$ and $s=\left[0, s_{x}, s_{y}, s_{z}\right]$, respectively. For the accelerometer, $e=[0,0,0,1]$ and $s=\left[0, f_{x}^{b}, f_{y}^{b}, f_{z}^{b}\right]$; for the magnetometer, $e=\left[0, b_{x}, 0, b_{z}\right]$ and $s=$ $\left[0, m_{x}^{b}, m_{y}^{b}, m_{z}^{b}\right] . q$ is the quaternion of the sensor pose, and its conversion relationship is as follows:

$$
s=q^{-1} e q
$$

The error equation is defined as:

$$
E(q)=q^{-1} e q-s
$$

The above equation is solved by Gauss Newton method:

$$
q_{t+1}=q_{t}-\mu_{t} \frac{\nabla E}{\| \nabla E||}
$$

where $\mu_{t}$ is the step size, and $\nabla$ is the differential operator. Combine Equation 24 and Equation 27,

$$
q_{t+1}=q_{t}+0.5 q_{t} \omega_{t} d_{t}-\beta \frac{\nabla E}{|| \nabla E||} d_{t}
$$

$0.5 q_{t} \omega_{t} d_{t}$ in Equation 28 is calculated and updated by the gyroscope. $\beta \frac{\nabla E}{\|\nabla E\| \mid} d_{t}$ is obtained by the accelerometer and magnetometer, and it is used to correct the angle error, where $\beta$ represents the error weight.

\subsection{Mahony AHRS}

For the Mahony algorithm, the current pose is also calculated in the form of quaternion using a gyroscope:

$$
q_{t+1}=q_{t}+0.5 q_{t} \omega_{t} d_{t}
$$

By calculating the difference of accelerometer as well as the difference of magnetometer between $n$-frame and $b$ frame, the error of pose is corrected.

$$
e=f^{b} \otimes v+m^{b} \otimes w
$$

$\otimes$ is vector cross-product. $e$ represents the relative rotation (error) between the measured inertial vector and the predicted vector. $v$ and $w$ are defined as follows:

$$
\begin{gathered}
v=\left[\begin{array}{l}
v_{x} \\
v_{y} \\
v_{z}
\end{array}\right]=C_{n}^{b}\left[\begin{array}{l}
0 \\
0 \\
1
\end{array}\right] \\
w=\left[\begin{array}{l}
w_{x} \\
w_{y} \\
w_{z}
\end{array}\right]=C_{n}^{b}\left[\begin{array}{c}
b_{x} \\
0 \\
b_{z}
\end{array}\right]
\end{gathered}
$$

After calculating $e$ by the proportion and integration regulator, the correction deviation $\delta$ regarding the gyroscope is obtained:

$$
\delta=K_{I} \int e d_{t}+K_{p} e
$$

The final corrected angular velocity is:

$$
\omega_{t}=\omega_{t}+\delta
$$

This equation is substituted into Equation 29 to update the pose calculation.

For the Mahony or Madgwick algorithm, the quaternion of its initial state can be calculated by the AHRS fundamental approach, and then its rotation matrix is converted to a quaternion. 


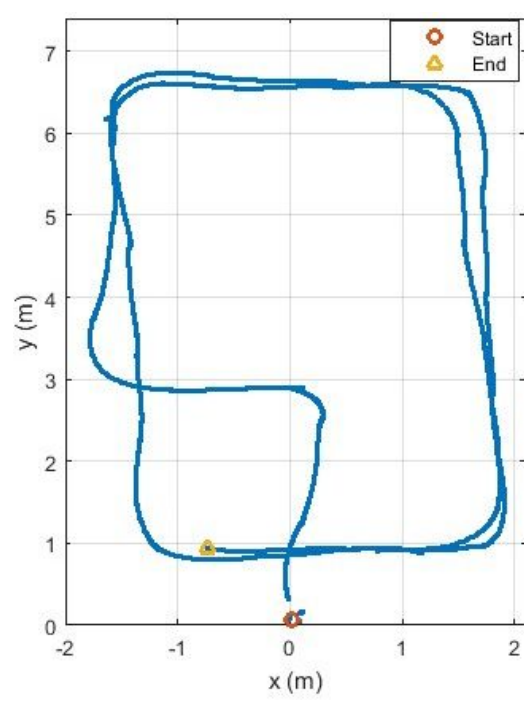

(a) Route1

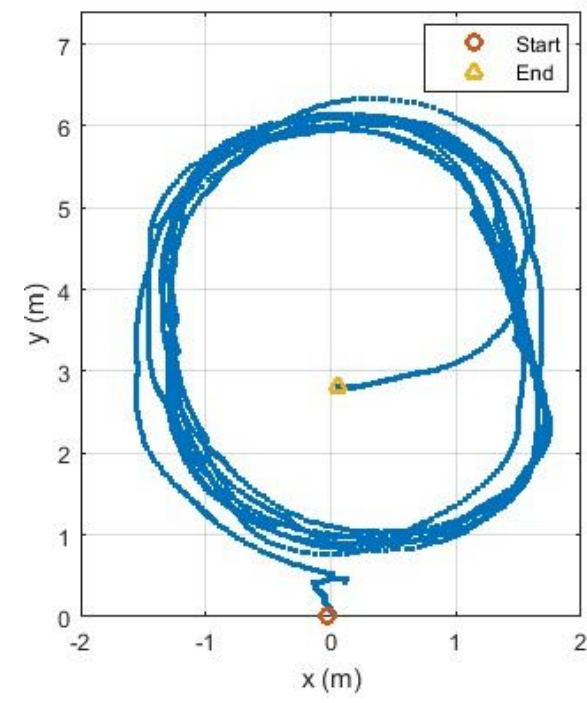

(b) Route2

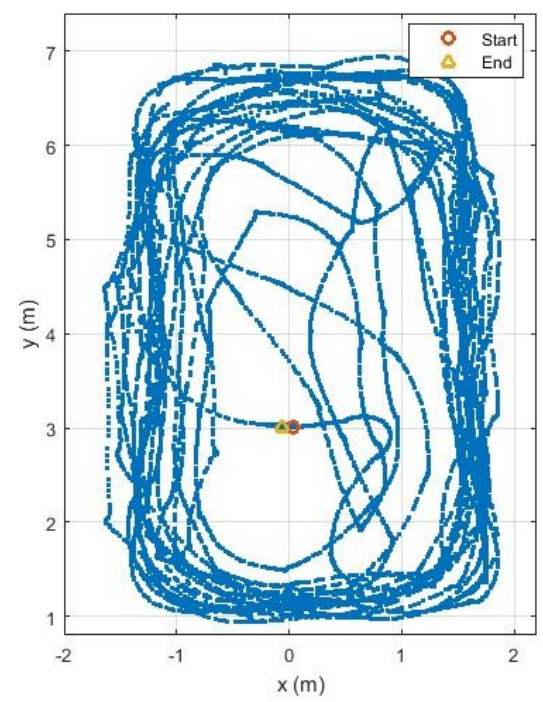

(c) Route3

Figure 3: Three experimental routes.

\section{Comparison of AHRS Algorithms with Foot-Mounted IMU}

We used the data published in [25] which provides high precision, ground-truth data obtained optical systems. A total of 16 sets of data are included in the data set, and the data are obtained from experiments conducted on different floor surfaces such as carpets and hard floors. A variety of walking modes are adopted in the experiments, including slow and fast walking, turns, loops, rectangles, rapid direction changes, walking backwards, running, and transitions. Among them, the first, eighth and fifteen sets of data are used as test objects, and their true trajectories are shown in Figure 3.

Route1 is a close-to-rectangular walking route with constant speed and less turning; Route 2 is an elliptical running route with more turns; Route3 is a more complex route with mixed walking and running. Due to the use of the public data set, calibration of magnetometer cannot be implemented and therefore no magnetometer is used in the AHRS algorithm.

The experimental design is carried out according to the following: Firstly, the more applicable algorithm for foot-mounted PDR, namely Madgwick algorithm, is selected by comparing it with the AHRS algorithm in the 9state CKF algorithm; then, the experiment shows that the directional accuracy of overall trajectory can be further improved by introducing the Madgwick algorithm in the 15state CKF algorithm; and finally, it is verified that the dy- namic setup of zero-speed detection threshold proposed in this paper can further improve the correction of directional errors.

\subsection{Analysis and comparison of Route1-2 positioning based on CKF under State-9}

The open source CKF algorithm under State-9 in [24] is adopted, and the related parameters are shown in Table 1. From the positioning results of Route1 and Route2 in Figure 4, the positioning trajectory obtained by AHRS fundamental algorithm (abbreviated as "Common") is poor since the drifts of accelerometer and gyroscope are not tracked in attitude calculation. The trajectory calculation using

Table 1: List of parameters.

\begin{tabular}{lc}
\hline Name & Value \\
\hline Time step & $1 / 100 \mathrm{~second}$ \\
Accelerometer noise & $0.01 \mathrm{~m} / \mathrm{s}^{2}$ \\
Gyroscope noise & $0.01 \mathrm{rad} / \mathrm{s}$ \\
Gravity & $9.8 \mathrm{~m} / \mathrm{s}^{2}$ \\
Zero-velocity(ZV) measurement noise & $0.01 \mathrm{~m} / \mathrm{s}$ \\
Gyroscope ZV detection threshold $\delta$ & $0.6 \mathrm{rad} / \mathrm{s}$ \\
A turning parameter $(\beta)$ of Madgwick's & 0.1 \\
filter & \\
A turning parameter $\left(K_{p} / K_{i}\right)$ of Ma- & $1 / 0$ \\
hony's filter & \\
\hline
\end{tabular}




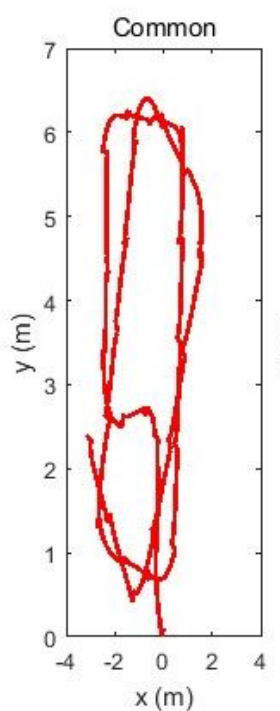

(a) Route1
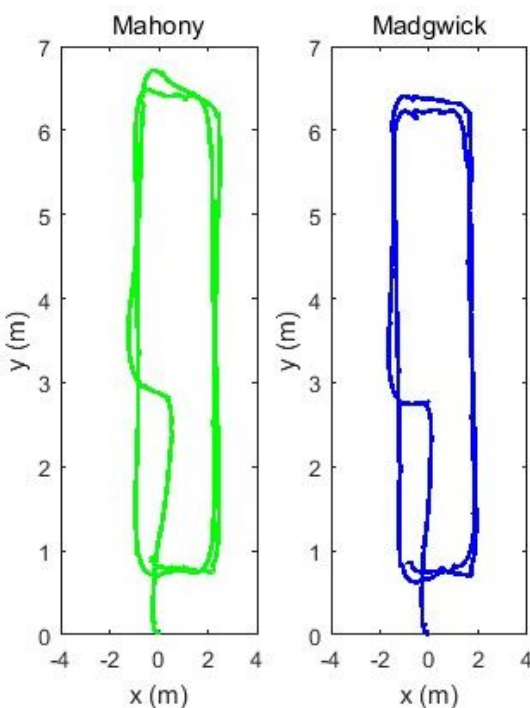
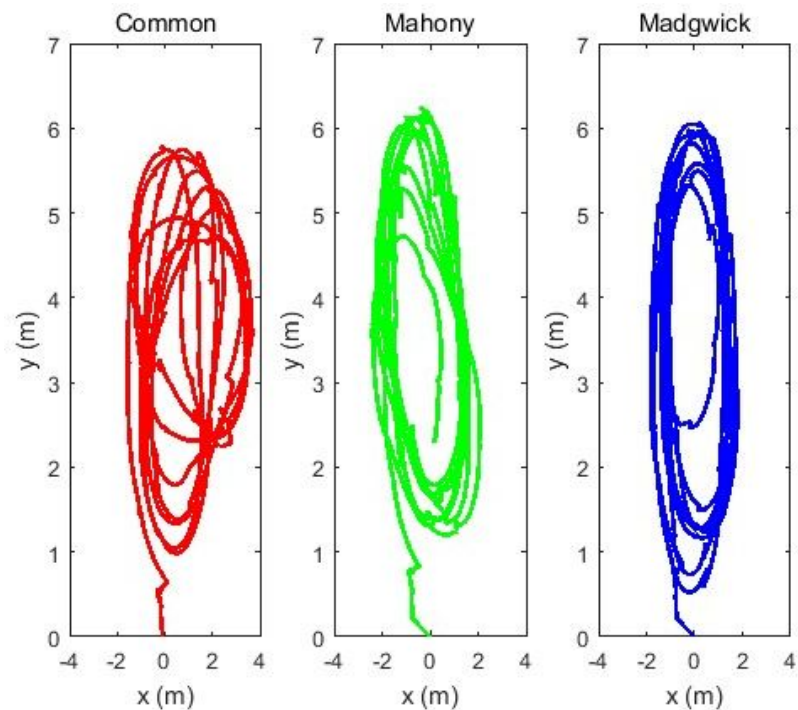

(b) Route2

Figure 4: Comparison of Route trajectories.
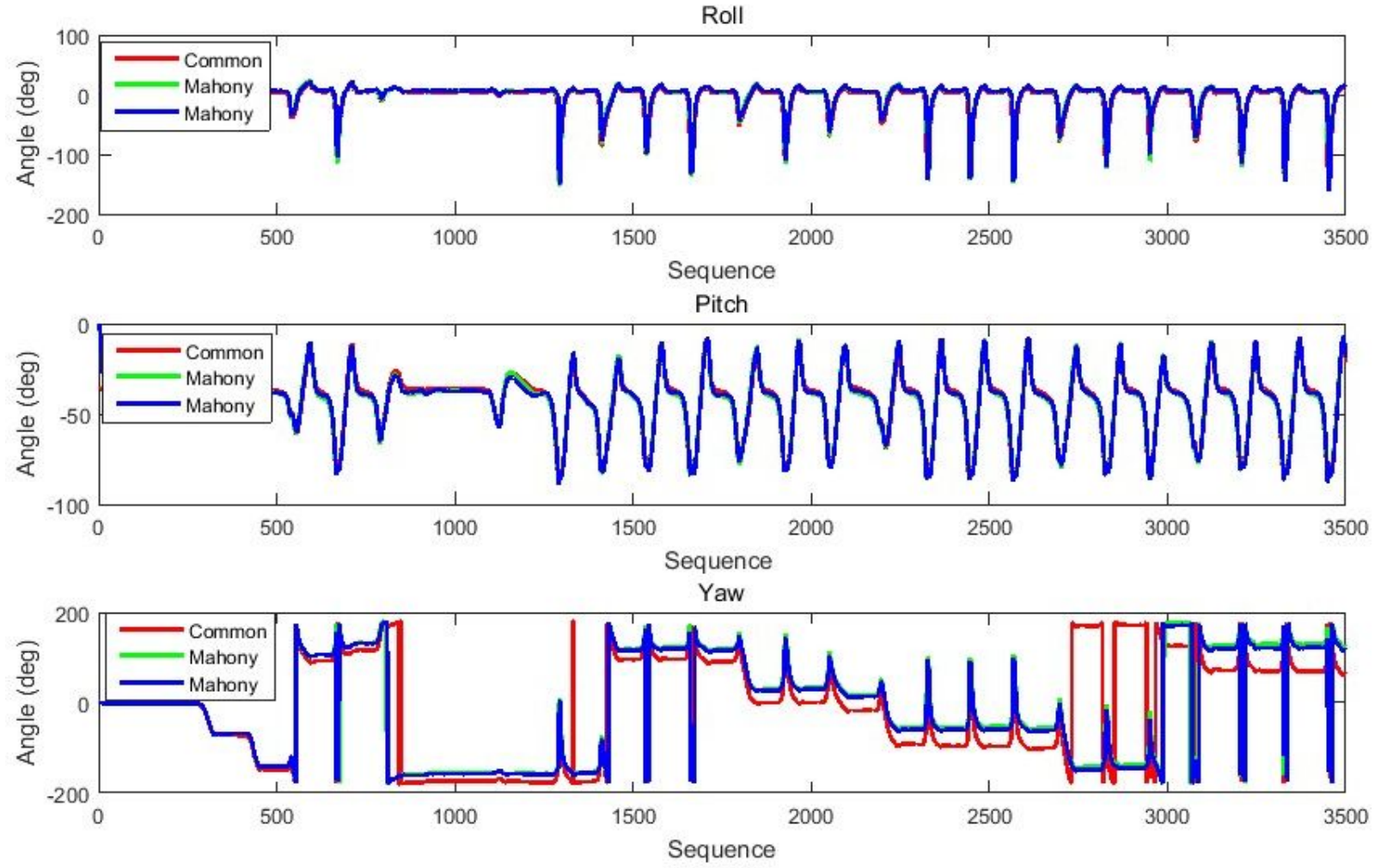

Figure 5: Comparison of heading angles of the three algorithms of Route1.

Mahony/Madgwick to conduct pose estimation is relatively accurate, and the result of Madgwick is the closest to the true trajectory.

The accuracy of the trajectory is inseparable from the accuracy of the direction, and the directional calculation results of the three algorithms are compared in Figure 5-6. It can be seen that for both Route 1 and Route 2, the differences of pitch and roll among the three calculation methods are not significant. This is mainly because the zerovelocity detection is periodically correcting the deviations 

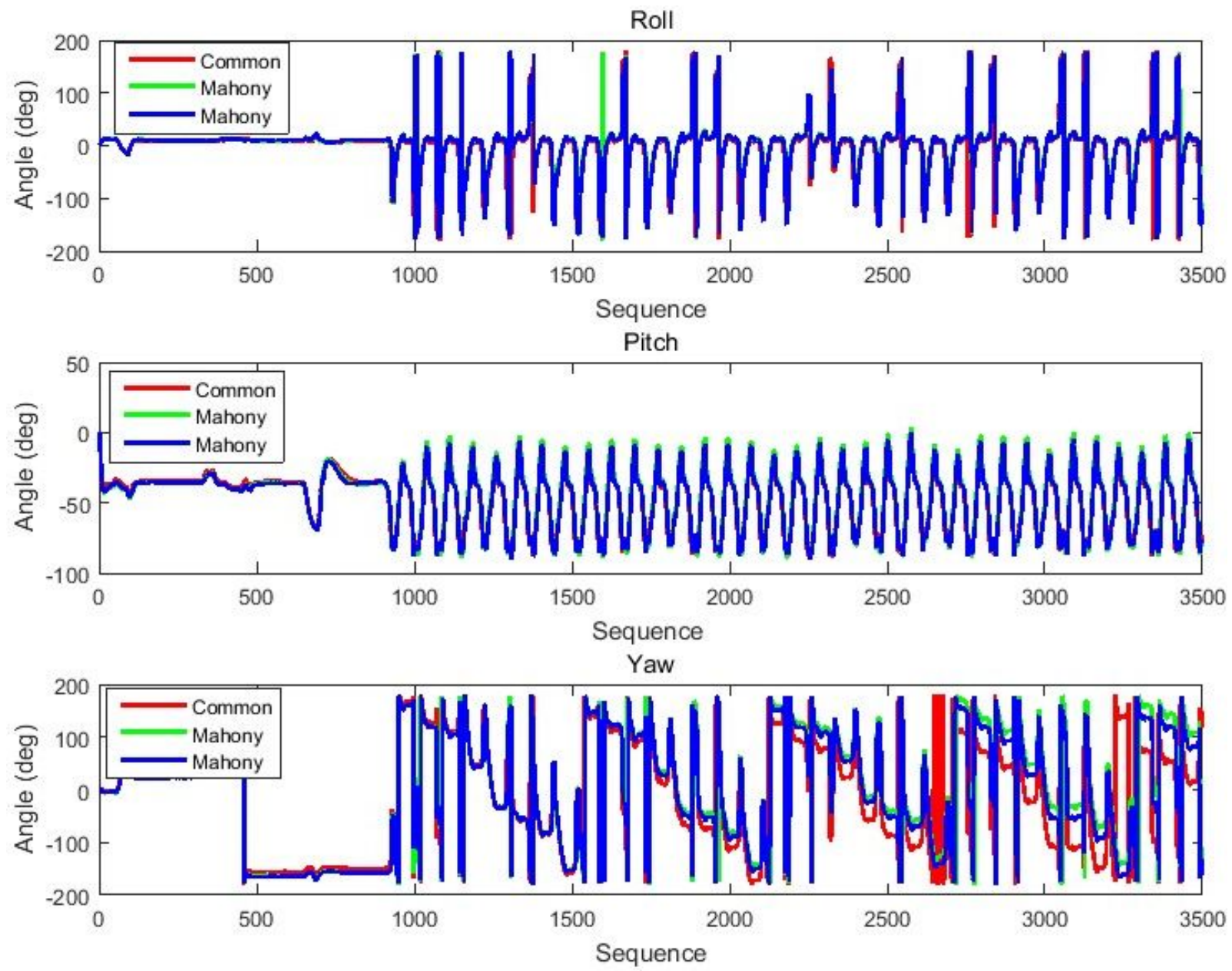

Figure 6: Comparison of heading angles of the three algorithms of Route2.

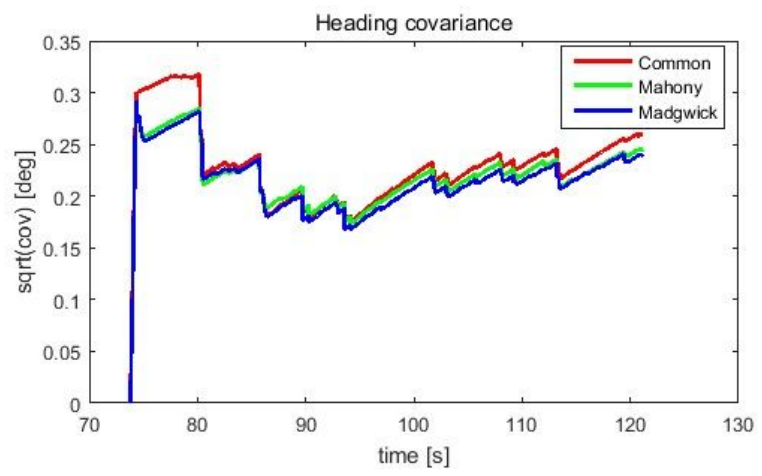

(a) Route1

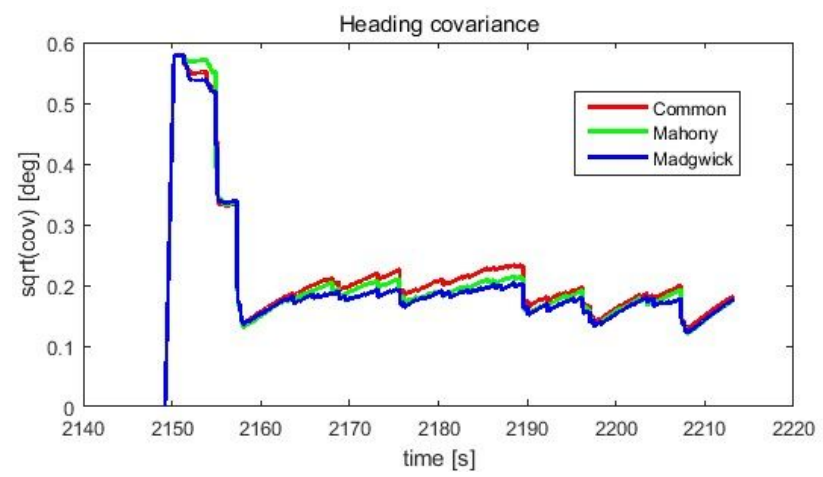

(b) Route2

Figure 7: Comparison of the covariance of heading angles.

of pitch and roll. For the yaw, the direction calculation of Madgwick algorithm is between that of Mahony and direct algorithm. Since there is no real direction for reference, from the results of the positioning trajectory, the calculation of the direction conducted by the Madgwick algorithm is the most accurate, which is also reflected in the comparison of the yaw covariance of the three algorithms in Figure 7. The covariance as well as the uncertainty of the Madgwick algorithm is the smallest. 


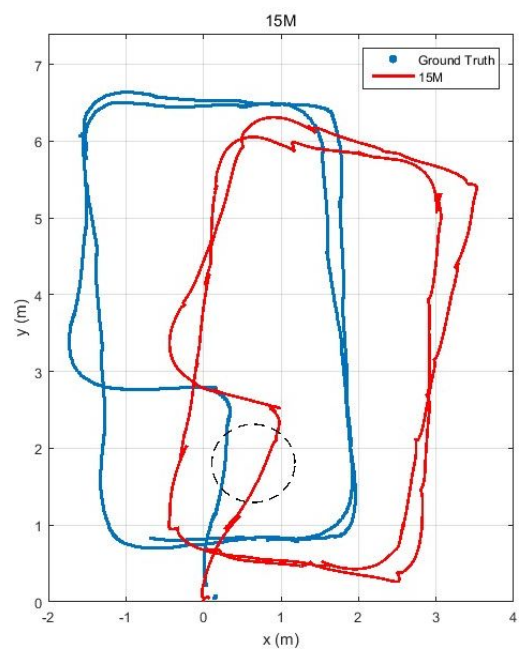

(a) $15 \mathrm{M}$

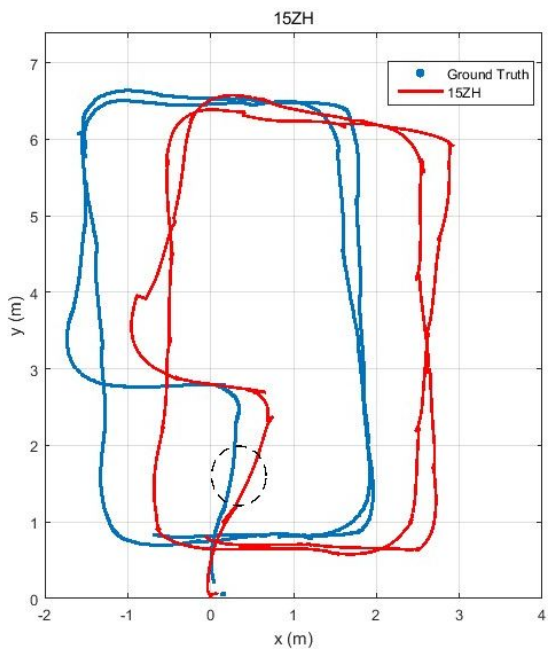

(b) $15 \mathrm{ZH}$

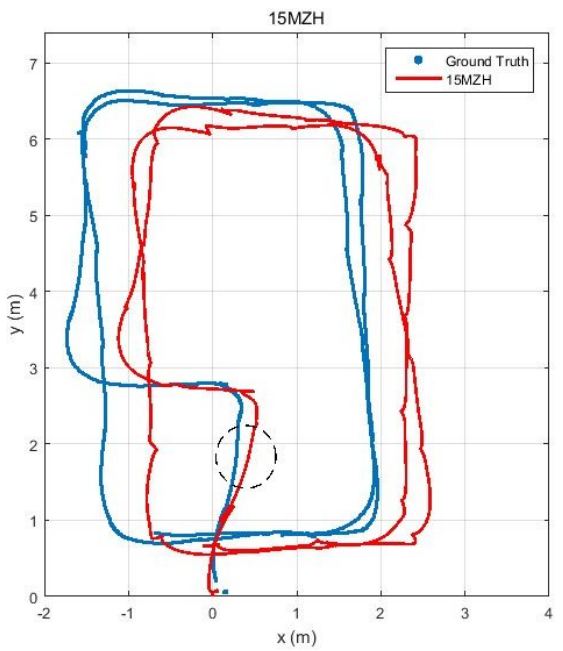

(c) $15 \mathrm{MZH}$

Figure 8: Comparison of the three AHRS algorithms of Route 1.

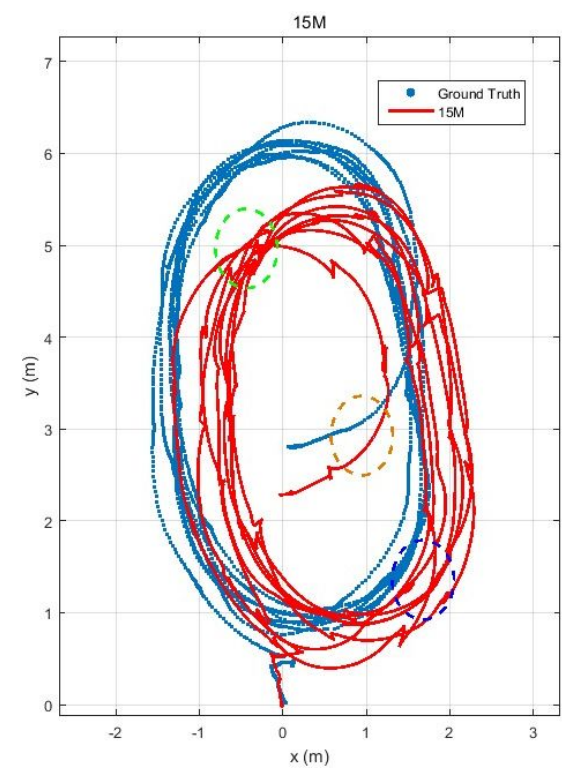

(a) $15 \mathrm{M}$

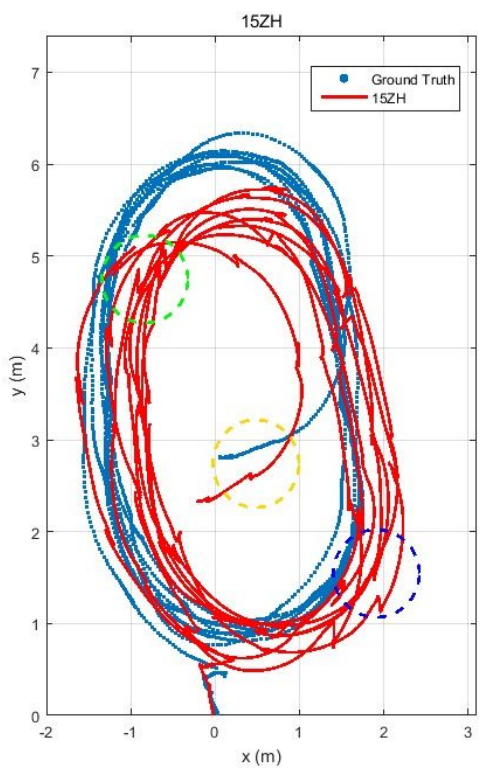

(b) $15 \mathrm{ZH}$

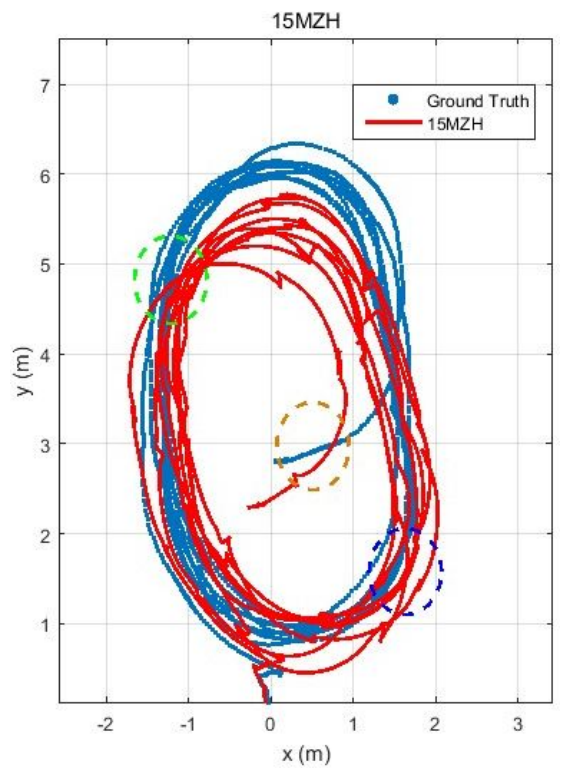

(c) $15 \mathrm{MZH}$

Figure 9: Comparison of the three AHRS algorithms of Route 2.

\subsection{Analysis and comparison of Route1-2 positioning based on CKF under State-15}

The performance of the AHRS algorithms depends strongly on the strategies used to reject perturbations, such as sudden accelerations or deformations of the Earth magnetic field, and the ability to estimate the biases of the gyroscopes. In the EKF under State-9, only the bias of position, velocity and angle are corrected, however, due to continuous integration of sensor noise and biases, the drift of gyroscope and accelerometer needs to be corrected too. Therefore, the CKF algorithm under State-15 [23] is referred, adding bias estimation of accelerometer and gyroscope to the state. The bias estimation is denoted as $X=$ $\left[\delta p^{n} \delta v^{n} \epsilon b_{g} b_{a}\right]$, where $b_{g}$ is the gyroscope bias, and $b_{a}$ is the acceleration bias. In addition, HDR and ZARU mentioned in [23] are introduced.

The positioning results of Route1-2 are shown in Figure 8-9. 15M represents CKF under State-15, and the Madgwick algorithm is used to calculate attitude; $15 \mathrm{ZH}$ repre- 
sents CKF under State-15, and the Common algorithm is used to calculate attitude, with HDR and ZARU as auxiliary; $15 \mathrm{MZH}$ represents that the Madgwick algorithm is used to calculate attitude, with HDR and ZARU as auxiliary. From the positioning results in Figure 8, the profiles of the positioning trajectory obtained by the three algorithms are basically the same, while considering the overall direction, the trajectory of $15 \mathrm{MZH}$ is closer to the real trajectory, as can be seen from the dashed circle in the figure. From the three dashed circles in Figure 9, the overall distribution of trajectory obtained from 15M and 15MZH based on the Madgwick algorithm is compacter than that of $15 \mathrm{ZH}$, and the trajectory obtained from $15 \mathrm{MZH}$ is closer to the true trajectory in the overall direction.

On the other hand, the positioning accuracy of the three algorithms can also be reflected by the positioning results on the Z-axis, as shown in Figure 10. Since the walking is on a plane, in theory, data on the Z-axis should be close to zero. From the positioning results of Route1 and Route2, it can be seen that the CKF algorithm with the introduction of Madgwick algorithm can reduce more positioning error to some extent.

\subsection{Adaptive zero-velocity detection algorithm}

The zero-velocity detection algorithm based on angular velocity proposed in [24] is adopted, and $\omega^{b}=\left[\omega_{x}^{b}, \omega_{y}^{b}, \omega_{z}^{b}\right]$ is the angular velocity under b-frame. The detection conditions are as follows:

$$
\begin{cases}\left\|\omega^{b}\right\|<\delta, & \text { still-phase } \\ \text { otherwise, } & \text { swing-phase }\end{cases}
$$

The norm value $\left\|\omega^{b}\right\|$ of angular velocity of Route1 and Route 2 are respectively shown in Figure 11. Route1 and Route2 are in walking state and running state, respectively. In the walking state with a constant velocity, the delay of the gait static state is longer, and the static state can be detected by a relatively small threshold $\delta_{1}$. However, in the running state with an increased velocity, the delay of the static state is relatively short, and part of the static state in a gait may be missed by $\delta_{1}$. When the sampling rate is low, it is possible that the overall static state in a gait is missed by $\delta_{1}$. These missed static states will reduce the constraints on velocity, resulting in larger positioning errors. However, it does not mean that the threshold can be enlarged. If the threshold is too large, such as $\delta_{2}$, the velocity is forced to zero before it reaches zero, causing the effective velocity value to fail to participate in the position integration which will also lead to positioning error.

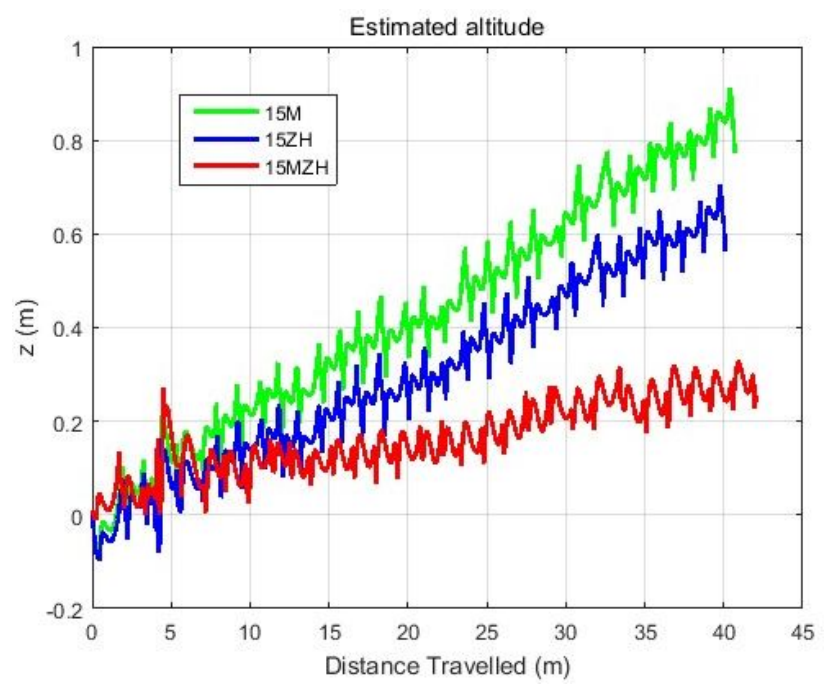

(a) Route1

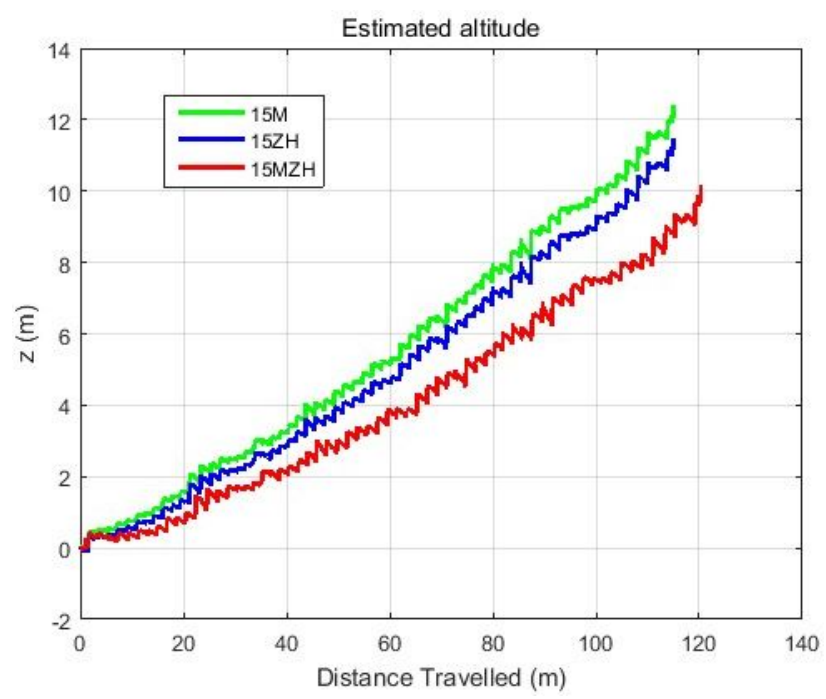

(b) Route2

Figure 10: Comparison of the errors of positioning height.

Route 3 corresponds to the mixed motion state. As shown in Figure 12, the part in the red circle and the parts outside represent the norm values of gyroscope in running state and non-running state, respectively. Obviously, it is difficult to use a fixed zero-velocity detection threshold at this time. The three-axis velocity of Route 3 is tracked by the CKF algorithm, as shown in Figure 13. The results in Figure 13 are obtained by a fixed zero-velocity detection threshold, and the change rule of the velocity can be roughly seen. The Route 3 experiences several stages, including walking, decelerating, running, decelerating and accelerating. Therefore, it is naturally conceivable to use velocity adaptation to set the zero-velocity detection threshold. 


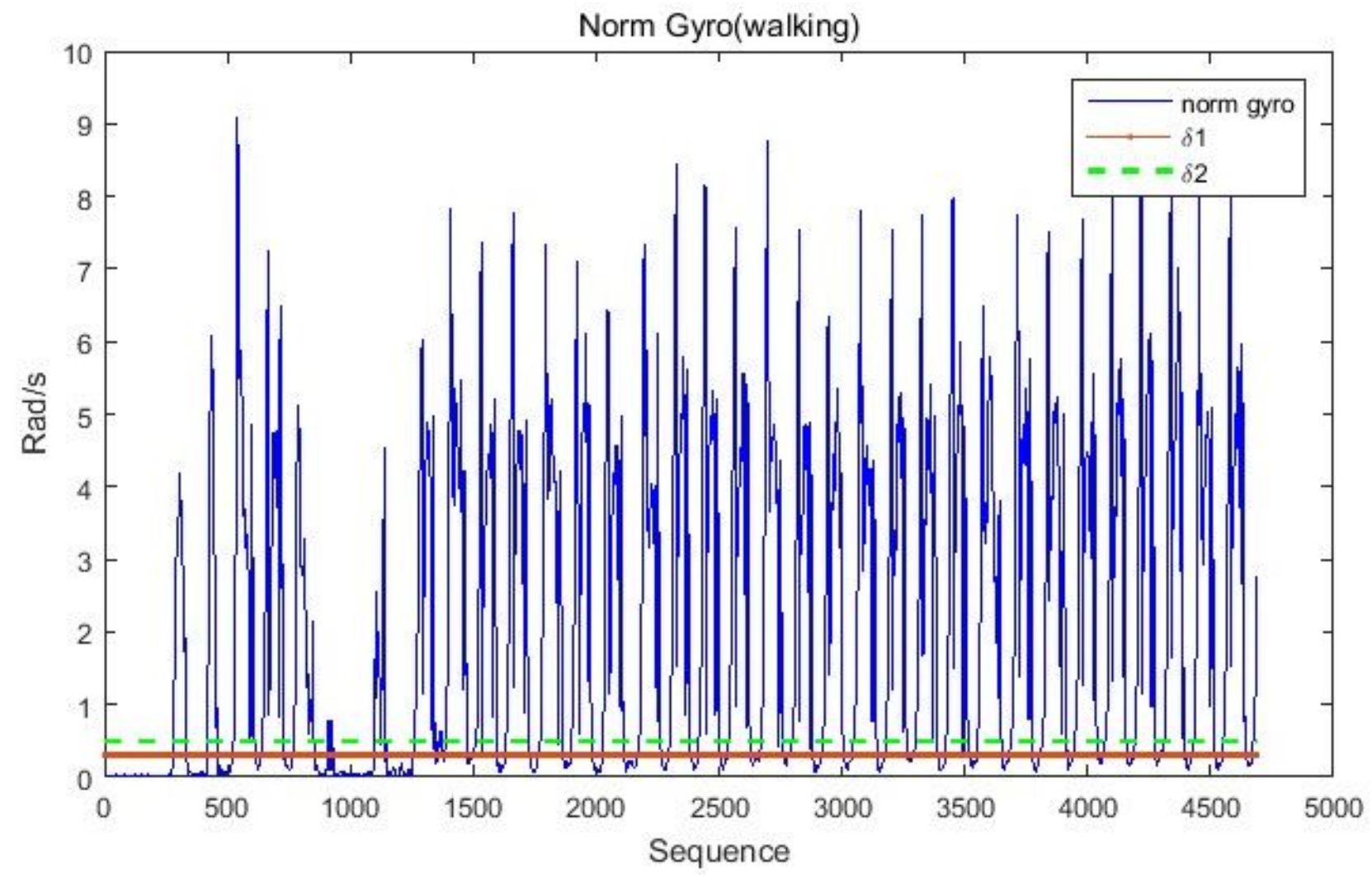

(a) Walking

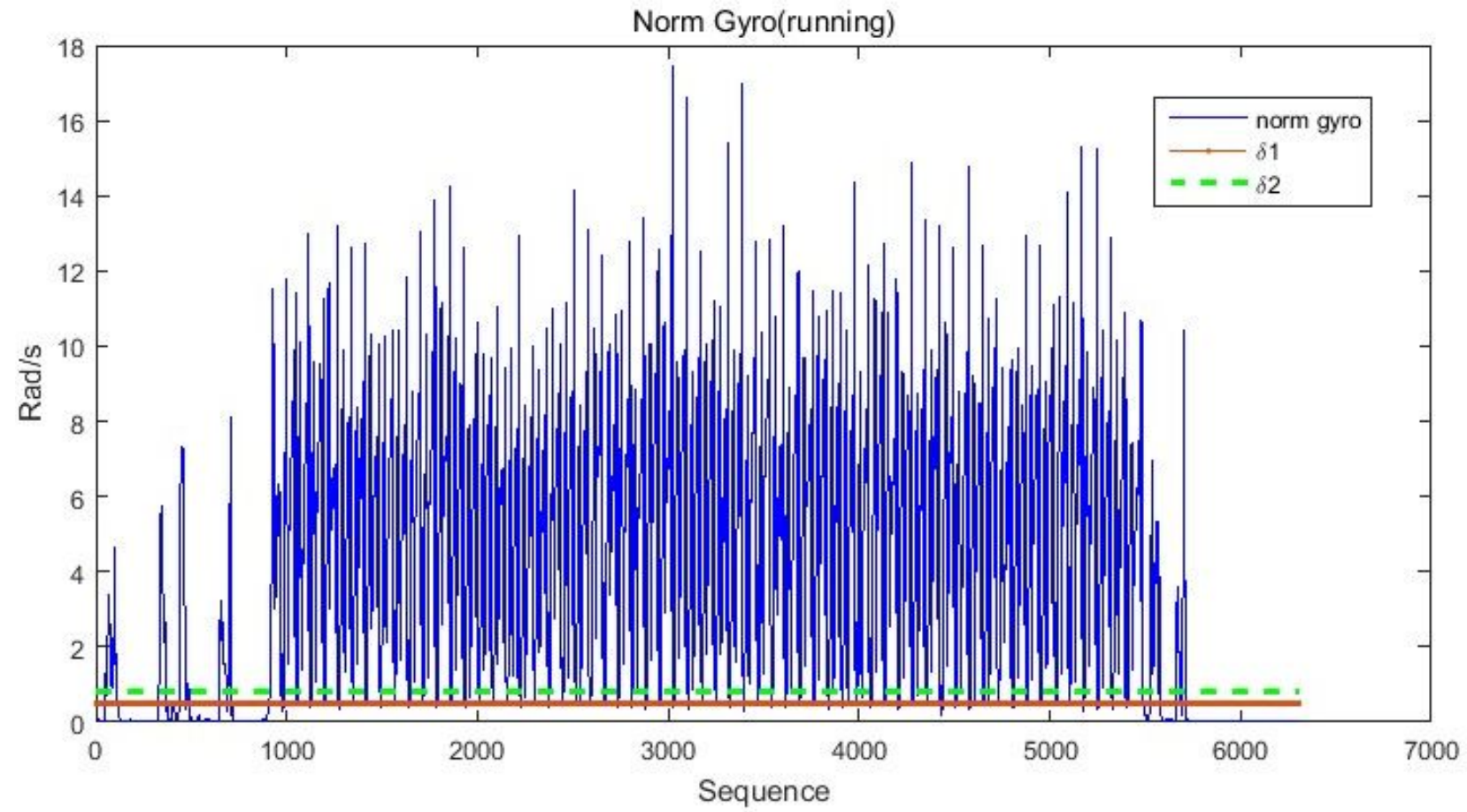

(b) Running

Figure 11: Norm values of Gyroscope of Route 1 and Route 2. 


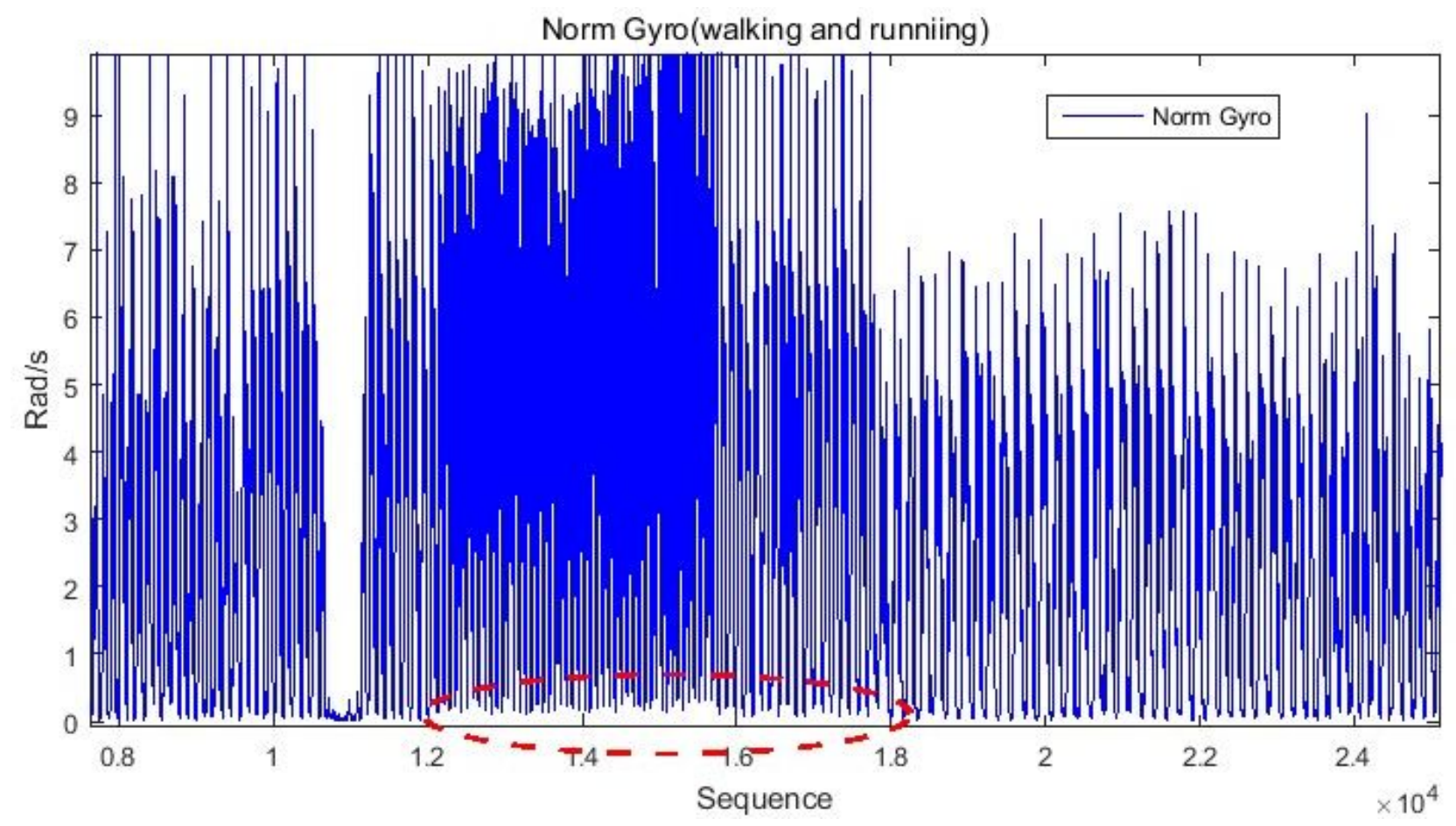

Figure 12: Norm value of Gyroscope of Route 3.

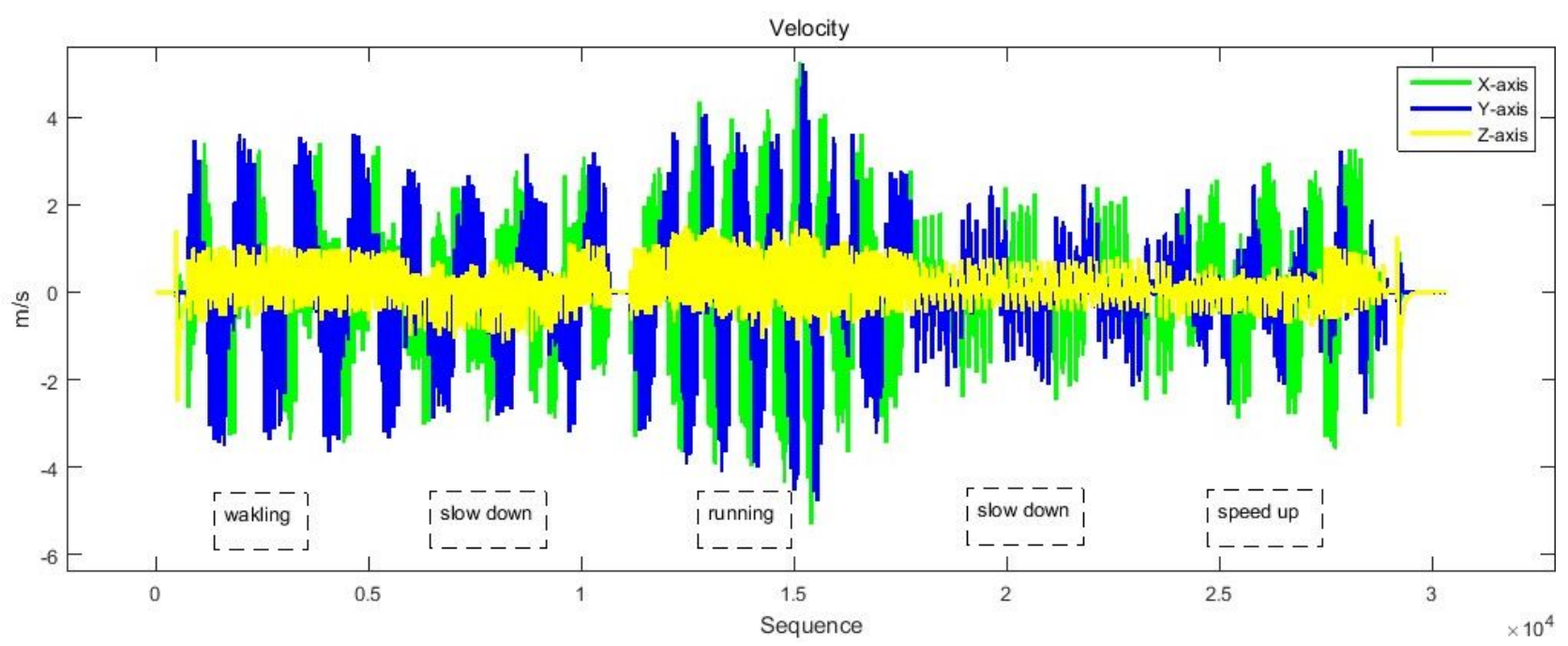

Figure 13: Three-axis velocity of Route3.

The extraction process of velocity feature is as follows. First, calculate the norm value of velocity, as shown in Figure 14. In order to determine the velocity state at a certain moment, the average velocity within 1 second can be calculated. The data sampling rate is $100 \mathrm{HZ}$ and the corresponding data length is 100 . Therefore, the window value $\mathrm{W}$ is equal to 100 , and the window value is used to calculate the average velocity of Route 3, as shown in Figure 15.
In order to make the state within the 1 second much clearer, the maximum velocity in this data window is calculated, as shown in Figure 16 and Equation 36. In Figure 17, the velocity above the red dashed line correspond to the running state, the parts between the red and green dashed lines correspond to the walking state, and the parts under the green dashed line correspond to the slow walking state. The corresponding zero-velocity detection 


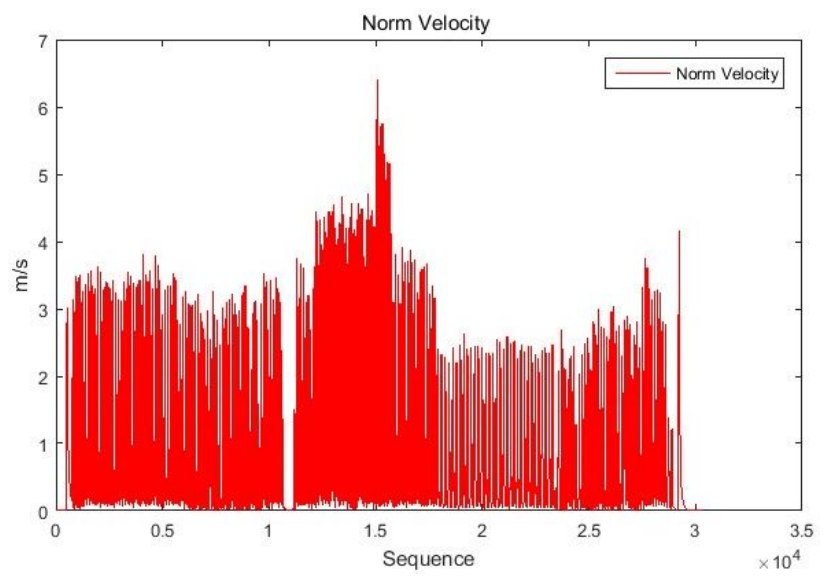

Figure 14: Norm value of velocity of Route 3.

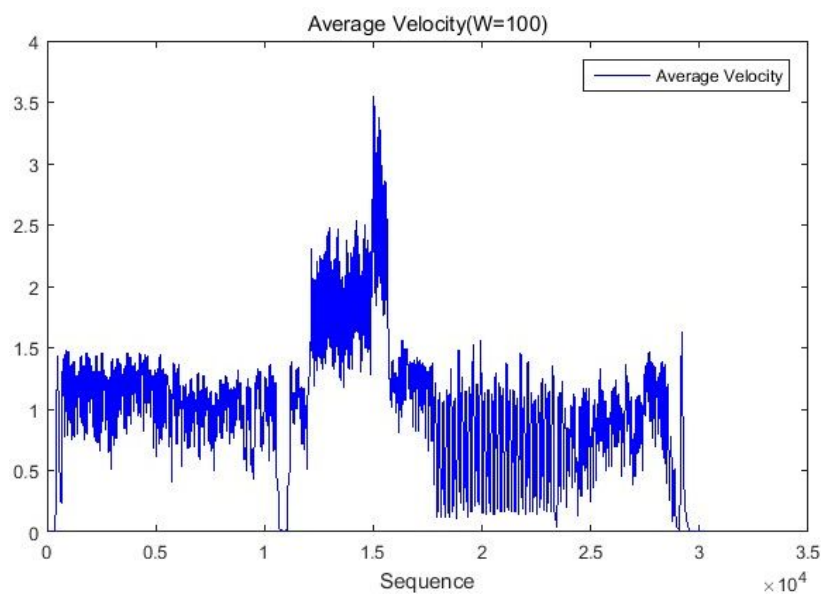

Figure 15: Average velocity of Route $3(W=100)$.

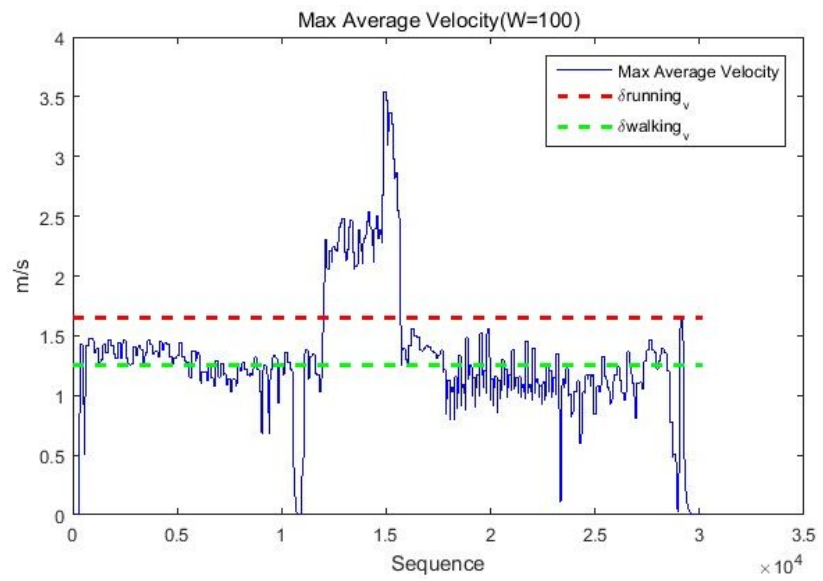

Figure 16: Maximum velocity in each data window of Route 3 $(W=100)$.

threshold $\delta$ for angular velocity can be set accordingly.

$$
\begin{cases}\bar{v}_{w} \geq \delta_{\text {running }}, & \text { running } \\ \delta_{\text {walking }} \leq \bar{v}_{w}<\delta_{\text {running }}, & \text { walking } \\ \bar{v}_{w}<\delta_{\text {walking }}, & \text { slow walking }\end{cases}
$$

Figure 17 shows the positioning trajectories of Route 3 obtained by fixed and adaptive zero-velocity detection threshold $\delta$ for angular velocity (algorithm denoted as 15MZHA). Obviously, the adaptive $\delta$ presents a more significant improvement on the positioning accuracy. This is because a more accurate correction of the zero-velocity moment can reduce the covariance of the direction and eliminate more errors of velocity and position, thereby improving the accuracy of the direction and positioning trajectory.

In the specific implementation of the algorithm, considering that the velocity is a real-time tracked variable, $\delta$ is initially given an empirical value, and then dynamically set with the velocity change.

\subsection{Test based on actual experimental data}

In order to better verify the proposed algorithm, the experimenter walks three times anticlockwise according to the route in Figure 18. The start and end points are shown in the top right corner, denoted by a triangle and a circle, respectively. The experimenter walks at normal speed in the first and third laps, and jogs in the second lap. The footmounted X-IMU is produced by X-IO Company in Britain, as shown in Figure 19.

The positioning results of 15MZH and 15MZHA are shown in Figure 20 and 21, respectively. In the overall trajectory, the positioning results of the two algorithms are basically consistent with the reference trajectory under the Madgwick algorithm and the assistive technologies of ZARU and HDR. However, compared with the first lap, the directional error of the last two laps gets bigger and bigger due to the inevitable accumulative error of MEMS-level IMU itself.

As for the 15MZH algorithm, when jogging in the second lap, since it cannot identify the change of pace, it still uses a fixed zero-speed threshold value to detect the zerospeed state, which will lead to the missing detection of many zero-speed states. These quiescent states that fail to be detected will reduce the constraint of speed, thus resulting in the much longer overall positioning trajectory. It can be known from the area within a broken circle in the left in Figure 20 that the trajectories of the last two laps almost overlap. Meanwhile, the missing detection of zero-speed states will reduce the correction of direction, and it can be seen from the area within a broken circle in the right in Figure 20 that the direction is abnormal in the second lap. 


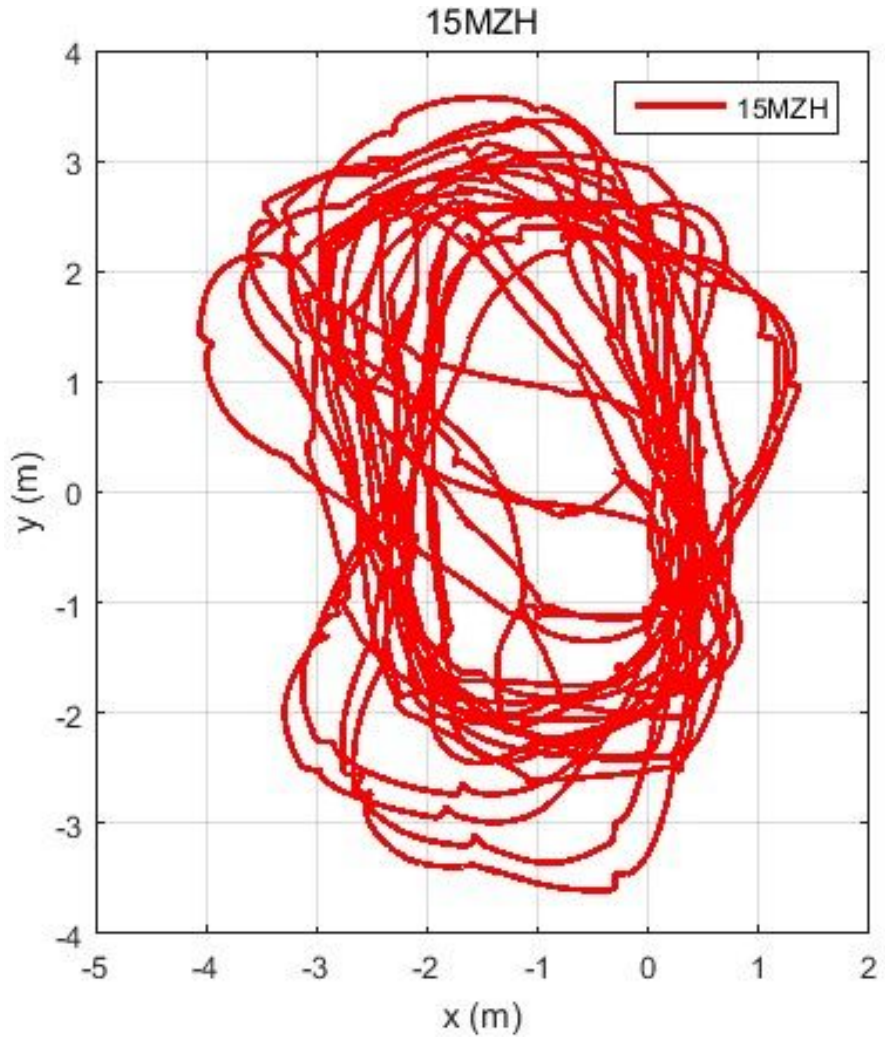

(a) Fixed $\delta$

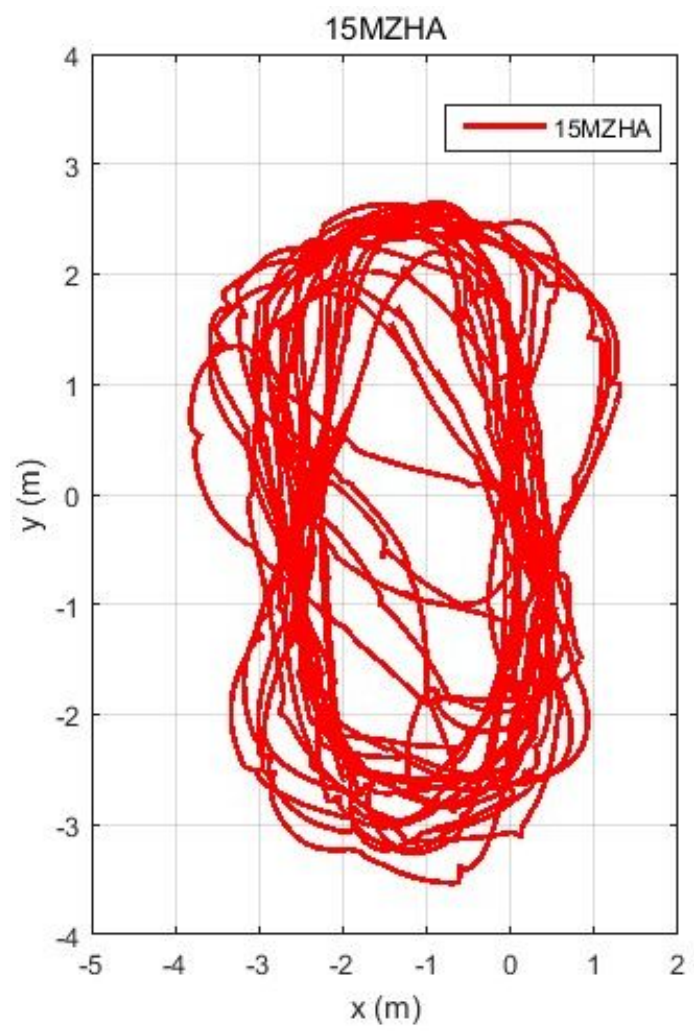

(b) Adaptive $\delta$

Figure 17: Comparison of different zero-velocity detection thresholds $\delta$ for angular velocity of Route 3 .

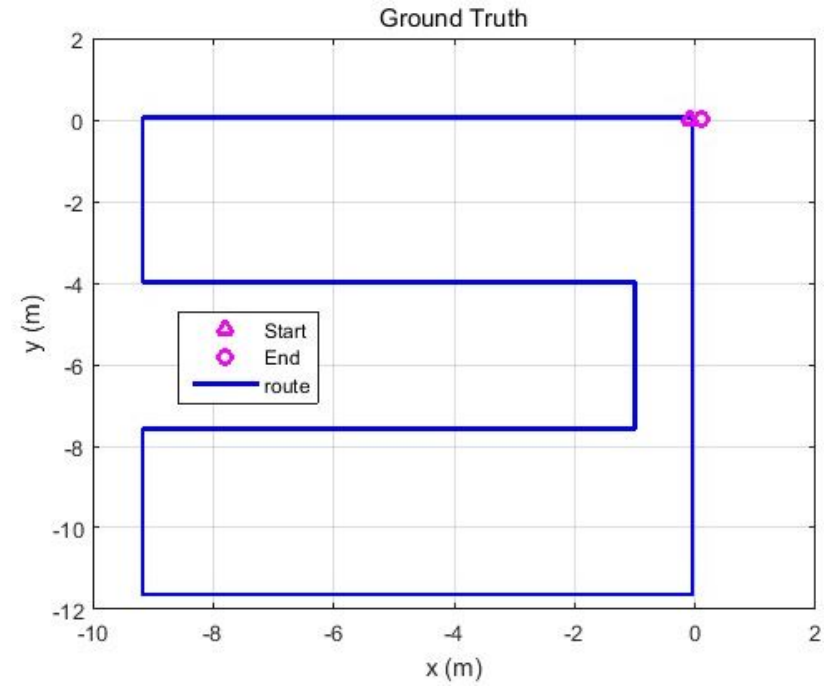

Figure 18: The design of the route in the experiment.

\section{Conclusions}

In this paper, the mainstream AHRS algorithms such as AHRS fundamental (Common), Mahony and Madgwick

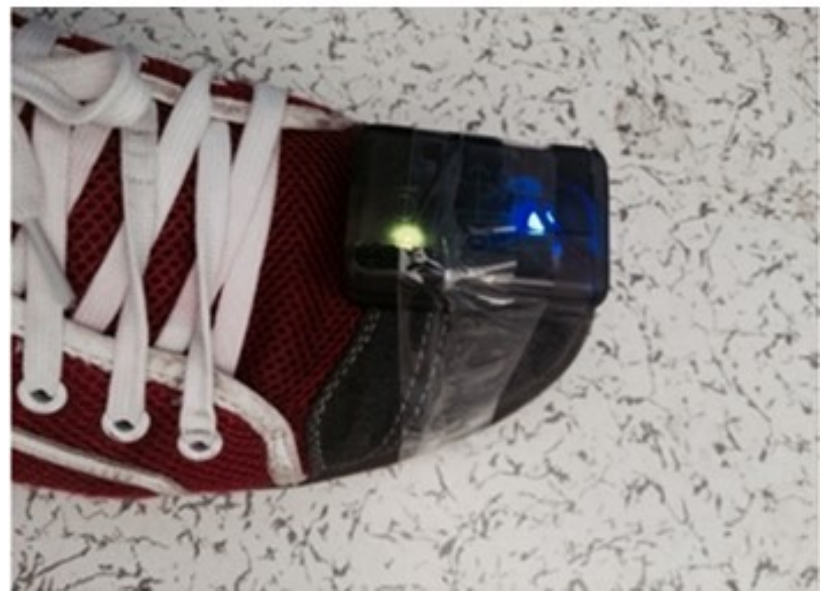

Figure 19: Foot-mounted IMU.

algorithms are introduced to the positioning algorithm based on foot-mounted IMU. The positioning accuracy of different AHRS algorithms adopting CKF algorithm under State-9 is compared and analysed, and it is also verified that the accuracy of the CKF algorithm under State-15 can be further improved by Madgwick algorithm. In order to further reduce the positioning error, a method of adap- 


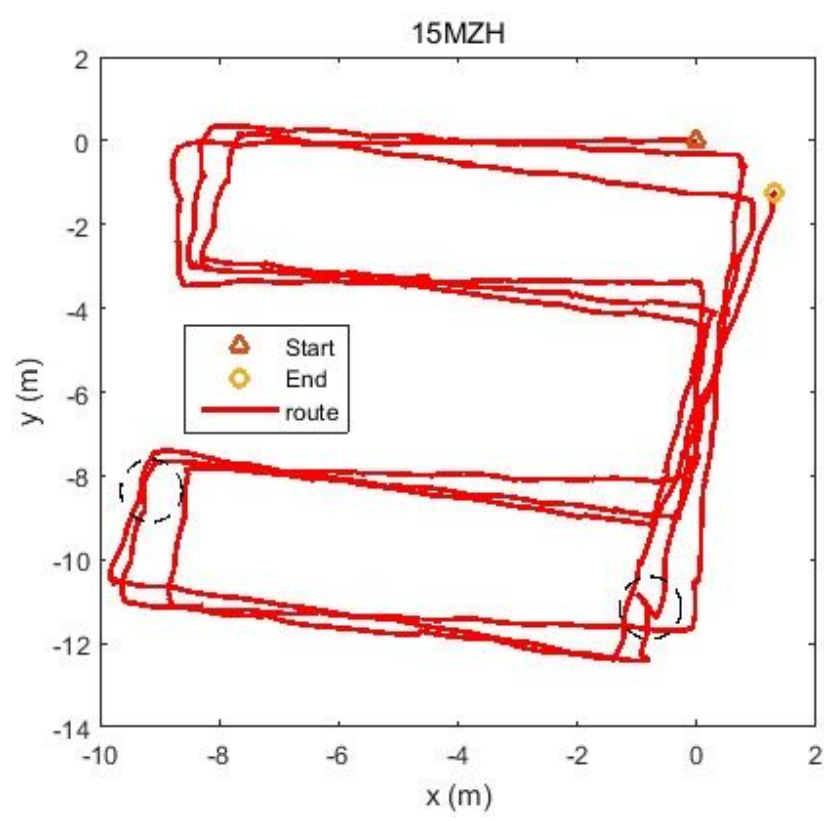

Figure 20: The positioning results of $15 \mathrm{MZH}$.

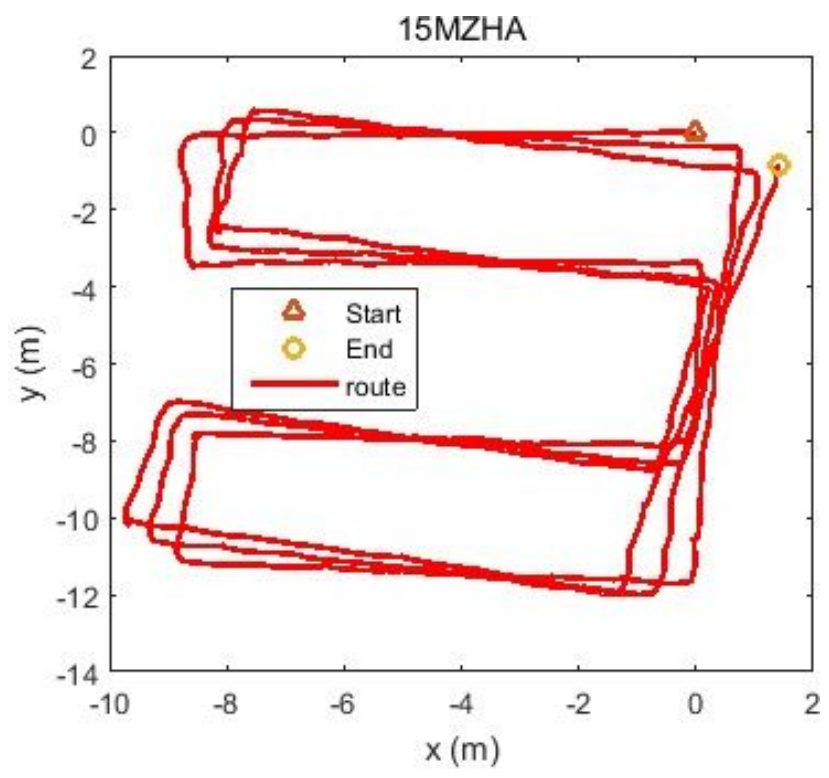

Figure 21: The positioning results of 15MZHA.

tively setting the zero-velocity detection threshold according to the velocity change is proposed, which improves the precision of the zero-velocity detection and direction accuracy. The future work will combine GNSS and UWB systems to realize stable, high-precision indoor and outdoor emergency positioning and map building system based on foot inertial navigation [26, 27].
Acknowledgement: This work was supported by the Fundamental Research Funds for the Central Universities under grant number $2017 \mathrm{XKQY020.}$

\section{References}

[1] Ilyas, M., Cho, K., Baeg, S. H., \& Park, S. (2016). Drift reduction in pedestrian navigation system by exploiting motion constraints and magnetic field. Sensors, 16(9).

[2] Romanovas, M., Goridko, V., Klingbeil, L., Bourouah, M., AlJawad, A., \& Traechtler, M., et al. (2013). Pedestrian Indoor Localization Using Foot Mounted Inertial Sensors in Combination with a Magnetometer, a Barometer and RFID. Progress in Location-Based Services. Springer Berlin Heidelberg.

[3] Norrdine, A., Kasmi, Z., \& Blankenbach, J. (2016). Step detection for zupt-aided inertial pedestrian navigation system using foot-mounted permanent magnet. IEEE Sensors Journal, 16(17), 6766-6773.

[4] Foxlin, E. (2005). Pedestrian tracking with shoe-mounted inertial sensors. IEEE Comput Graph Appl, 25(6), 38-46.

[5] Krach, B., \& Robertson, P. (2008). Integration of foot-mounted inertial sensors into a Bayesian location estimation framework. The Workshop on Positioning (Vol.3, pp.55-61). IEEE.

[6] Robertson, P., Angermann, M., \& Krach, B. (2009). Simultaneous localization and mapping for pedestrians using only footmounted inertial sensors. International Conference on Ubiquitous Computing (pp.93-96). ACM.

[7] Robertson, P., Angermann, M., \& Khider, M. (2010). Improving simultaneous localization and mapping for pedestrian navigation and automatic mapping of buildings by using online human-based feature labeling., 2010(2010-12-27), 365-374.

[8] Bruno, L., \& Robertson, P. (2011). WiSLAM: Improving FootSLAM with WiFi. International Conference on Indoor Positioning and Indoor Navigation (pp.1-10). IEEE.

[9] Puyol, M. G., Robertson, P., \& Angermann, M. (2013). Managing large-scale mapping and localization for pedestrians using inertial sensors. IEEE International Conference on Pervasive Computing and Communications Workshops (pp.121-126). IEEE.

[10] Nilsson, J. O., Skog, I., Handel, P., \& Hari, K. V. S. (2012). Footmounted INS for everybody-an open-source embedded implementation. Position Location and Navigation Symposium (Vol.1, pp.140-145). IEEE.

[11] Nilsson, J. O., Skog, I., \& Händel, P. (2012). A note on the limitations of zupts and the implications on sensor error modeling. Signal Processing.

[12] Nilsson J O, Händel P. (2013).Standing still with inertial navigation. International Conference on Indoor Positioning and Indoor Navigation (IPIN), 28th-31th October 2013.

[13] Nilsson, J. O., Zachariah, D., Skog, I., \& Händel, P. (2013). Cooperative localization by dual foot-mounted inertial sensors and inter-agent ranging. Eurasip Journal on Advances in Signal Processing, 2013(1), 164.

[14] Skog, I., Nilsson, J. O., \& Handel, P. (2014). Pedestrian tracking using an IMU array. IEEE International Conference on Electronics, Computing and Communication Technologies (pp.1-4). IEEE.

[15] Skog, I., Nilsson, J. O., Händel, P., \& Nehorai, A. (2016). Inertial sensor arrays, maximum likelihood, and cramér-rao bound. 
IEEE Transactions on Signal Processing, 64(16), 4218-4227.

[16] Puyol, M. G., Robertson, P., \& Heirich, O. (2012). Complexityreduced FootSLAM for indoor pedestrian navigation. International Conference on Indoor Positioning and Indoor Navigation (Vol.7, pp.1-10). IEEE.

[17] Johnson, M. E., \& Sathyan, T. (2011). Improved orientation estimation in complex environments using low-cost inertial sensors. Proceedings of the, International Conference on Information Fusion (pp.1 - 8). IEEE.

[18] Kaiser, S., Puyol, M. G., \& Robertson, P. (2012). Maps-based angular PDFs used as prior maps for FootSLAM. Position Location and Navigation Symposium (pp.113-119). IEEE.

[19] Mahony, R., Hamel, T., \& Pflimlin, J. M. (2008). Nonlinear complementary filters on the special orthogonal group. IEEE Transactions on Automatic Control, 53(5), 1203-1218.

[20] Madgwick, S. O. H., Harrison, A. J. L., \& Vaidyanathan, R. (2011). Estimation of IMU and MARG orientation using a gradient descent algorithm. IEEE International Conference on Rehabilitation Robotics(Vol.2011, pp.1-7). IEEE.

[21] O. Nilsson(2008). Navigation System for a Micro-UAV. Master thesis, KTH, Stock-holm, Sweden.
[22] S. O. H. Madgwick, R. Vaidyanathan, and A. J. L. Harrison. An efficient orientation filter for inertial measurement units (IMUs) and magnetic angular rate and gravity (MARG) sensor arrays. Department of Mechanical Engineering, Tech. Rep., April 2010.

[23] Jiménez, A. R., Seco, F., Prieto, J. C., \& Guevara, J. (2010). Indoor pedestrian navigation using an INS/EKF framework for yaw drift reduction and a foot-mounted IMU. Positioning Navigation and Communication(pp.135-143). IEEE.

[24] Fischer, C., Sukumar, P. T., \& Hazas, M. (2013). Tutorial: implementing a pedestrian tracker using inertial sensors. IEEE Pervasive Computing, 12(2), 17-27.

[25] Angermann, M., Robertson, P., Kemptner, T., \& Khider, M. (2010). A high precision reference data set for pedestrian navigation using foot-mounted inertial sensors. International Conference on Indoor Positioning and Indoor Navigation (pp.1-6). IEEE.

[26] Piórkowski, A. (2018). Construction of a dynamic arrival time coverage map for emergency medical services. Open Geosciences, 10(1), 167-173.

[27] Bogusz, J., Gruszczynski, M., Figurski, M., \& Klos, A. (2015). Spatio-temporal filtering for determination of common mode error in regional GNSS networks. Open Geosciences, 7(1), 140148. 